\title{
Deciphering the genomic structure, function and evolution of carotenogenesis related phytoene synthases in grasses
}

\author{
Bianca Dibari ${ }^{1,3}$, Florent Murat ${ }^{1}$, Audrey Chosson ${ }^{1}$, Véronique Gautier ${ }^{1}$, Charles Poncet ${ }^{1}$, Philippe Lecomte ${ }^{1}$, \\ Ingrid Mercier ${ }^{2}$, Hélène Bergès ${ }^{2}$, Caroline Pont ${ }^{1}$, Antonio Blanco ${ }^{3}$ and Jérôme Salse ${ }^{1 *}$
}

\begin{abstract}
Background: Carotenoids are isoprenoid pigments, essential for photosynthesis and photoprotection in plants. The enzyme phytoene synthase (PSY) plays an essential role in mediating condensation of two geranylgeranyl diphosphate molecules, the first committed step in carotenogenesis. PSY are nuclear enzymes encoded by a small gene family consisting of three paralogous genes (PSY1-3) that have been widely characterized in rice, maize and sorghum.

Results: In wheat, for which yellow pigment content is extremely important for flour colour, only PSY1 has been extensively studied because of its association with QTLs reported for yellow pigment whereas PSY2 has been partially characterized. Here, we report the isolation of bread wheat PSY3 genes from a Renan BAC library using Brachypodium as a model genome for the Triticeae to develop Conserved Orthologous Set markers prior to gene cloning and sequencing. Wheat PSY3 homoeologous genes were sequenced and annotated, unravelling their novel structure associated with intron-loss events and consequent exonic fusions. A wheat PSY3 promoter region was also investigated for the presence of cis-acting elements involved in the response to abscisic acid (ABA), since carotenoids also play an important role as precursors of signalling molecules devoted to plant development and biotic/abiotic stress responses. Expression of wheat PSYs in leaves and roots was investigated during ABA treatment to confirm the up-regulation of PSY3 during abiotic stress.
\end{abstract}

Conclusions: We investigated the structural and functional determinisms of PSY genes in wheat. More generally, among eudicots and monocots, the PSY gene family was found to be associated with differences in gene copy numbers, allowing us to propose an evolutionary model for the entire PSY gene family in Grasses.

Keywords: Carotenoids, Phytoene synthase, Wheat, Intron loss, Abiotic stress, Evolution

\section{Background}

Carotenoids represent a diverse group of pigments found in bacteria, fungi and plants [1]. In plants, carotenoids have several important functions, essential for plant development as: $(i)$ they serve as accessory pigments to harvest light for photosynthesis and constitute the basic structural units of photosynthetic apparatus and (ii) they also act as photoprotectors for plants to adapt to high light stress [2]. In addition, oxidative cleavage of carotenoids produces apocarotenoids considered as $(i)$ signals in plant development, $(i i)$

\footnotetext{
* Correspondence: jsalse@clermont.inra.fr

'INRA - UMR 1095 'Génétique Diversité Ecophysiologie des Céréales' (GDEC),

5 Chemin de Beaulieu, 63100, Clermont-Ferrand, France

Full list of author information is available at the end of the article
}

antifungal agents, (iii) contributors to flower and fruit flavours and aromas [3]. The phyto-hormone abscisic acid, the most well known apocarotenoid, plays an important role in plant response to stress conditions [3]. In addition, carotenoids have long been considered as essential nutrients as well as important health beneficial compounds. In animals including humans, unable to synthesize carotenoids de novo, dietary carotenoids are essential precursors of vitamin A and retinoid compounds needed in development $[4,5]$.

Plant carotenogenesis occurs in the membrane of chloroplasts, chromoplasts and amyloplasts, plastids having different internal membrane architectures. The enzymes involved in the carotenoid biosynthetic pathway are

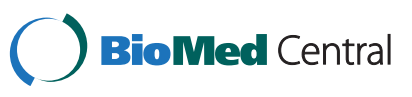


nuclear encoded and targeted to the plastids. Carotenoid biosynthesis begins with the formation of the 40-carbon backbone, phytoene, a step mediated by phytoene synthase (PSY) ( $c f$ Figure 1) [6,7]. PSY, which is known to control carotenoid flux in seeds and to catalyze the first committed step in carotenogenesis, is thought to be a rate-limiting enzyme. In "Golden Rice" [8], for example, PSY activity was crucial to obtain increased carotenoid levels [9], whereas bacterial carotene desaturase CrtI had no effect [10]. PSY is coded by a small gene family, which had been already shown to exist throughout the Grasses [5] and which consists of three paralogous genes that have been identified and characterized in rice, maize and sorghum. The three rice PSY genes share the same structure, with six exons and five introns, and they exhibit a tissuespecific expression. Rice endosperm is carotenoid free and transcripts for all three genes are absent. In photosynthetic tissues, all three PSY mRNAs are present, but with different levels of expression, OsPSY1 and OsPSY2 playing predominant roles with similar expression patterns and regulation in response to light. OsPSY3 plays a specialized role as it is not regulated by light but is strongly inducible in roots by high salt concentrations and/or drought.
OsPSY1 and OsPSY2 contain cis-acting elements involved in light regulation which are absent from the promoter region of OsPSY3, explaining why PSY3 does not respond to light [11]. Li et al. [12] described the PSY gene family and their tissue-specific transcript patterns in maize. They showed that PSY1 is the only gene family member for which transcript level increased during maize endosperm development and was correlated with accumulation of endosperm carotenoids. Transcript changes of PSYs were monitored in maize leaves during de-etiolation. In darkgrown plants, PSY1 represented the major transcript whereas $P S Y 2$ was the only paralog for which transcript levels increased in response to illumination, suggesting then that PSY2 plays an important role in controlling leaf carotenogenesis during greening. The abundance of PSY3 in roots suggests that it plays a unique role in roots carotenogenesis when induced by drought, salt and exogenous ABA treatment. The increase of $P S Y 3$ transcripts observed was associated with an induced level of carotenoid intermediates, and elevation of other downstream carotenogenic genes, followed by ABA accumulation [12].

PSY genes are of primary importance in wheat due to their impact on flour colour, so that numerous genetics

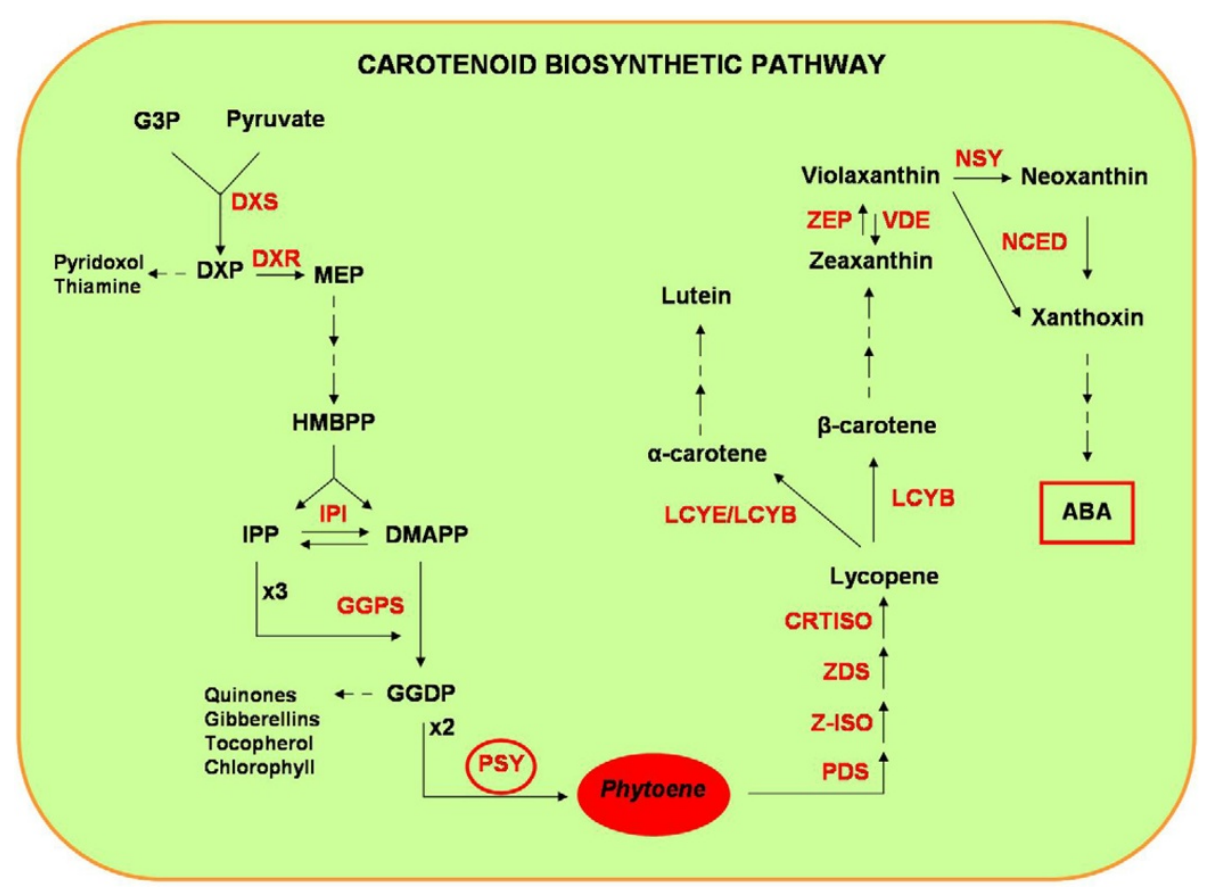

Figure 1 Carotenoid biosynthetic pathway (adapted from Lu et al. 2008). The isopentenyl diphosphate (IPP) is synthesized via the 2-Cmethyl-D-erythritol 4-phosphate (MEP) pathway. IPP and DMAPP form the central intermediate geranylgeranyl diphosphate (GGDP) for carotenoid biosynthetic pathway. Phytoene synthase (PSY) acts at the condensation of two molecules of GGDP to form the phytoene, the first step of the carotenoid pathway. Names of compounds are highlighted in black and of enzymes in red. Abbreviations: G3P, glyceraldheyde-3-phosphate; DXP, 1-deoxy-D-xylulose 5-phosphate; HMBPP, 1-hydroxy-2-methyl-2-butenyl 4-diphosphate; ABA, abscisic acid; DXR, 1-deoxy-D-xylulose 5-phosphate reductoisomerase; DXS, 1-deoxy-D-xylulose 5-phosphate; IPI, isopentenyl diphosphate isomerase; GGPS, geranylgeranyl diphosphate synthase; PDS, phytoene desaturase; Z-ISO, Z-carotene isomerase; ZDS, Z-carotene desaturase; CRTISO, carotene isomerase; LCYB, $\beta$-cyclase; LCYE, $\epsilon$-Cyclase; ZEP, zeaxanthin epoxidase; VDE, violaxanthin de-epoxidase; NSY, neoxanthin synthase; NCED, 9-cis-epoxycarotenoid dioxygenase. 
studies have been conducted on yellow pigment (YP) content in wheat grain [13]. A high YP concentration is a desirable trait in durum wheat and is the target of breeding programs worldwide [14]. However, low YP is a breeding target in hexaploid wheat, for which bright white flour is preferred. The inheritance of YP is complex, controlled by additive gene effects and highly heritable [15]. Several QTLs for YP have been identified in both durum and bread wheat on chromosomes 1A [16], 1B [17,18], 3A [19], 3B [16,20], $4 \mathrm{~A}$ and $5 \mathrm{~A}$ [21], 2A, 4B and 6B [13], 5B [16], and 6A [17]. However, the majority of studies agreed that chromosome group 7 has the greatest impact in controlling YP content in bread wheat [13,16-19,22-24]. Elouafi et al. [22] identified two minor QTLs on chromosome 7A and a third major QTL on chromosome 7BL. This last QTL has been corroborated in two other durum wheat populations $[13,24]$ and is ortholog to the one identified in hexaploid wheat on the same chromosome [25]. A QTL for YP in durum wheat was identified by Patil et al. [16] on chromosome 7A that accounted for up to $55 \%$ of the trait variation. Mares and Campbell [20] identified QTLs on chromosome 7A in two hexaploid wheat populations that had a large impact on flour yellowness, and correspond to loci also associated with xanthophyll concentration. Cloning of genes controlling flour pigmentation would be of great help in breeding for genotypes with appropriate levels of yellow pigments in durum and bread wheat. Using a PCR-based approach Cenci et al. [26] identified and mapped clones from a durum wheat BAC library thought to contain genes coding for important enzymes involved in carotenogenesis. In their study, putative phytoene synthase, phytoene desaturase and $\zeta$-carotene desaturase clones were assigned to the chromosome groups 5, 4 and 2, respectively. To date, four PSY genes have been identified in durum wheat, forming two paralogous series on chromosome groups 5 (Psy2) and 7 (Psy1) [13]. One of these, Psy1-B1, co-segregates with a QTL for YP on 7BL $[13,24]$. Psy1-A1 was localized on the distal end of the chromosome 7AL in one hexaploid wheat mapping population, associated with variation for flour colour [18]. Wheat PSY1 genomic sequences show similar exon-intron characteristic to PSY1 known in other Grass species, with six exons separated by five introns [18]. A partial sequence of PSY2 was also obtained and it was mapped on the short arm of the chromosome group 5 [13], with no clear data regarding the impact of $P S Y 2$ on carotenoid accumulation.

In this article, we report evidence that the wheat PSY gene family also consists of three paralogous genes and that PSY3 is characterized by a novel gene structure, consequence of intron loss events. Since the wheat $P S Y 3$ promoter region is characterized by cis-acting elements involved in plant response to abiotic stress, we studied changes in wheat PSY expression levels in leaves and roots during exogenous treatment with ABA. Finally, after comparative genomic investigation of PSY loci, we reconstructed the paleo-history for this gene family in Grasses identifying evolutionary events that have shaped PSY gene copies in modern species.

\section{Results}

\section{PSY3 gene sequencing and mapping in bread wheat}

We recently reassessed the syntenic relationships within monocots (rice, maize, Brachypodium, sorghum and Triticeae) and developed tools to identify precisely chromosometo-chromosome orthologous relationships and derived Conserved Orthologous Set (COS) markers, [27,28]. Rice (LOC_Os09g38320), sorghum (Sb02g032370) and Brachypodium (Bradi4g37520.1) PSY3 orthologs, available from the literature, respectively mapped on chromosomes 9, 2 and 4 were used to clone wheat PSY genes. On the basis of the known rice/sorghum/wheat/Brachypodium syntenic blocks covering PSY3 orthologs, PSY3 was expected to be located on chromosome group 5 in wheat (Figure 2a, [29]). The blast search did not result in significant orthologous wheat sequences from public databanks (either EST database, [30] or the genome sequence database [31]). Primers were therefore developed from conserved genic regions characterized between rice and Brachypodium PSY3 coding sequences to screen a bread wheat BAC library ( $T$. aestivum cultivar 'Renan' [32], see Materials and Methods). This library is organised in 384-well plates of BAC pools with a specific screening strategy to reduce the number of PCR reactions necessary to identify a single clone of interest (see Materials). Primer pairs PSY_4g37520.1_bra_F2/PSY_437520.1_bra_R2, designed on the fourth exon of Brachypodium PSY3 (Additional file 1: Table S1) were used for library screening. Positive pools were sequenced and three homoeoforms were obtained and assigned to the wheat chromosomes using nullisomic-tetrasomic lines [33]. By crossing the pool coordinates (plates/lines/columns) we identified the BAC clones harbouring PSY3 gene homoeologs. Three BAC clones spanning the $P S Y 3$ gene of $\mathrm{A}, \mathrm{B}$ and $\mathrm{D}$ sub-genomes of $T$. aestivum were screened with PCR-specific markers targeting each of the three PSY3 homoeologs (Additional file 1: Table S1 and Figure 2b-c) and then sequenced. Sequencing resulted in 138 391, 149199 and $130149 \mathrm{bp}$ of gapped contigs for, respectively, the A, B and D sub-genomes. Each homoeologous copy was then precisely assigned to chromosome bins using genome-specific primer pairs (Additional file 1: Table S1) and DNA from deletion lines: PSY3s were located precisely on the chromosome bins 5AL10-0.57-0.78, 5BL14-0.75-0.76 and 5DL1-0.60-0.74, respectively, for $\mathrm{A}, \mathrm{B}$ and $\mathrm{D}$ genome copies.

\section{Identification and characterization of PSY3 loci}

BAC sequencing and annotation ( $c f$ Materials and Methods) made it possible to identify PSY3 orthologous regions. We selected ungapped contigs of $25000 \mathrm{bp}, 53411 \mathrm{bp}$ and 46 


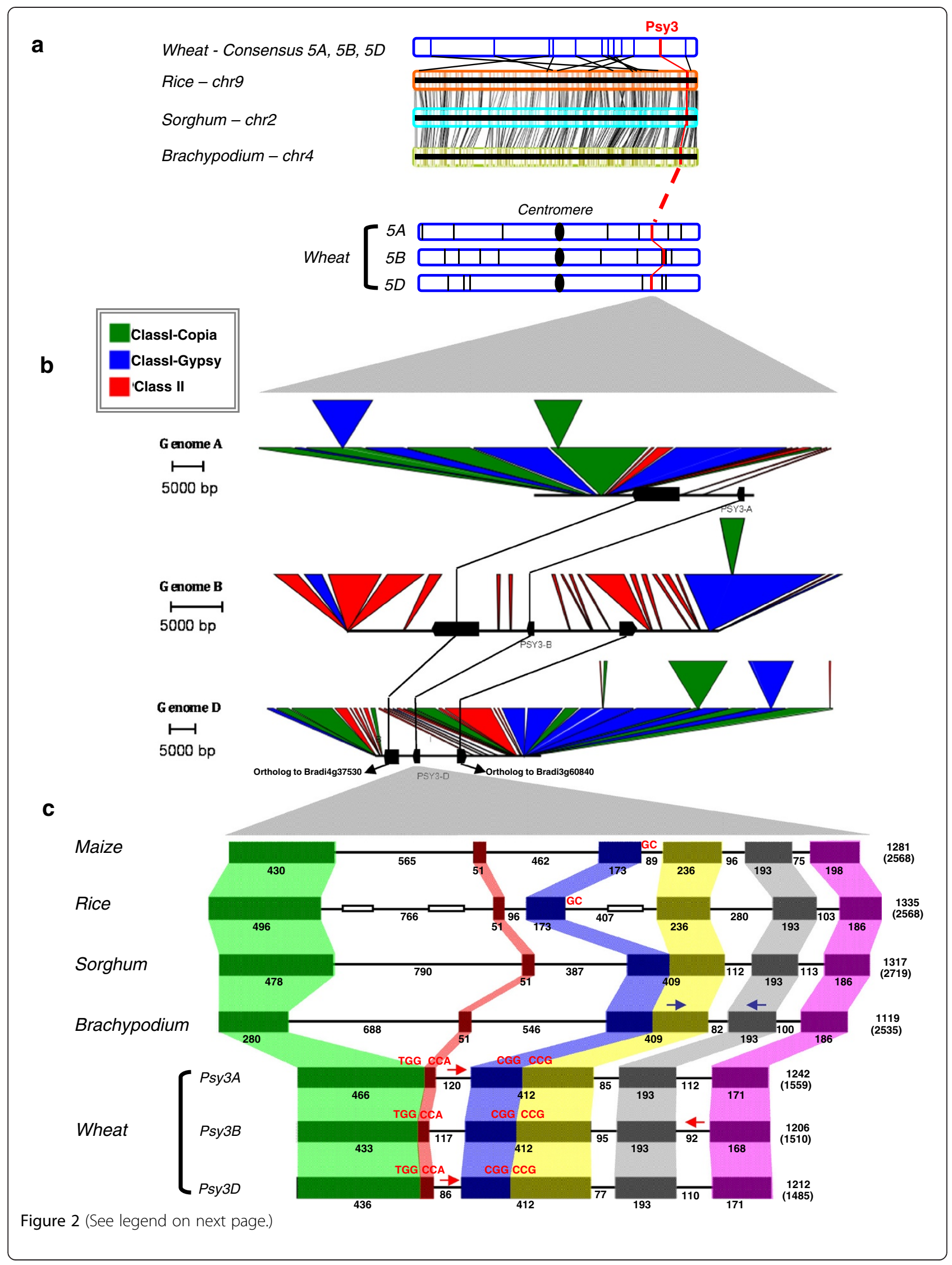




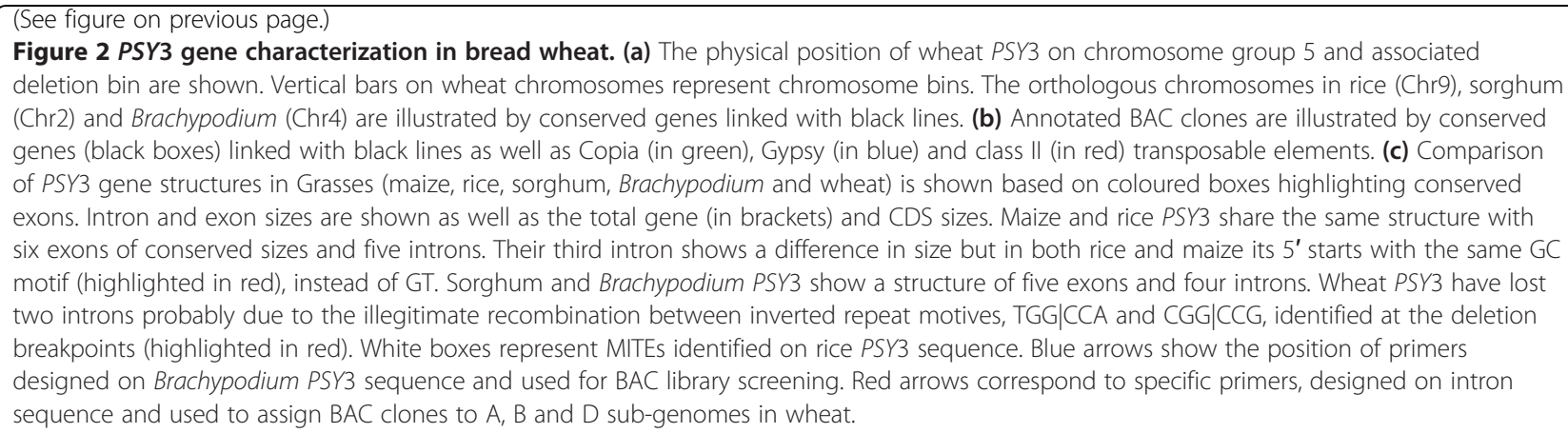

803 bp harbouring, respectively, PSY3A, PSY3B and PSY3D genes (Additional file 1: S1, S2, S3). Figure 2b illustrates BAC annotation results as well as micro-colinearity with rice, Brachypodium, maize and sorghum genome sequences, confirming that PSY3 and Bradi4g37530.1 genes are at orthologous positions. In the PSY3 loci we found the insertion of a non-syntenic gene (orthologous to Bradi3g60840). According to paleogenomic data in monocots [29], this gene is located on the syntenic chromosomes 2, 4 and 3 of, rice, sorghum and Brachypodium genomes respectively. In wheat, this gene would be expected to map on the orthologous chromosome group 6 and not group 5, where it was found based on the PSY sequenced region assignation. We can thus suppose that this gene has been specifically transposed in wheat from an ancestral donor region (group 6) to the modern current acceptor region (group 5).

Alignment with Brachypodium PSY3 CDS clarified wheat PSY3s exon-intron boundaries. Wheat PSY3s showed a structure characterized by four exons spaced by three introns that were numbered according to their position within the genes, with the first intron closest to the $5^{\prime}$ and the third intron closest to the 3 ' end (Figure 2c). This structure and exon-intron sizes were conserved between the A, $\mathrm{B}$ and $\mathrm{D}$ homoeologous copies. Rice and maize PSY3 were the only genes with six exons spaced by five introns. In Brachypodium and sorghum, the loss of the third intron determined the appearance of a different structure. In rice and maize, this intron showed at its donor site, the T/C transition, starting with the GC motif instead of GT. We also found in rice PSY3 the presence of Miniature Inverted Transposable Elements (MITEs), in the first intron: nt628-nt741 and nt953-nt1130, and in the third intron (nt1769-nt1955) the same MITE was identified in inverted orientation. Wheat PSY3 was found to include the largest first exon of $466 \mathrm{bp}$, $433 \mathrm{bp}$ and $436 \mathrm{bp}$, respectively, for PSY3A, B and D, resulting from fusion of the first and second exons after an intron loss event. The second exon had $412 \mathrm{bp}$ and resulted from the fusion of the third and fourth exons. It was characterized by a close sequence homology with the third exon of the Brachypodium ortholog. Figure 3a shows PSY3 gene structure evolution throughout Grasses. Rice and maize share the same PSY3 gene structure, while sorghum and Brachypodium gene structures are characterized, as in wheat, by the loss of the third intron and the subsequent fusion of the two flanking exons. We suggest that, during evolution, different and independent intron loss events occurred. At the deletion breakpoints, we identified repeated inversed motifs, TGG| CCA and CGG|CCG (Figure 3b, c) in the first and the second exon respectively. It is therefore possible to assume that these motifs have facilitated intron loss events through illegitimate recombination. The first intron lost was at phase 1 , while the second was at phase 0 . The TSSP database (see materials) defined the promoter region of PSY 3 , around $2 \mathrm{~Kb}$ upstream from the start codon and PolyA Signal Miner software identified the terminator signal 865 bp, 867 bp and 828 bp, respectively for A, B and D genomes. Sequence analysis allowed us to identify a duplication of the fourth exon in the PSY3D locus, $1729 \mathrm{bp}$ downstream from the stop codon. This repeat did not start with the trinucleotide GTG, as expected based on the exon \#4 sequence (but with the complementary nucleotides $\mathrm{CAC}$ ), and was interrupted by a $154 \mathrm{bp}$ deletion flanked at each extremity by the TACTGG motif. We can thus suppose that a novel illegitimate recombination event mediated the duplication of the fourth exon and its insertion in the sequence.

Despite intron/exon shuffling events reported previously, it was possible to predict protein structure. PSY3A encodes a protein of 413 amino acids, longer than those coded by PSY3B and PSY3D homoeologs (401 and 403 amino acids, respectively) (Additional file 1: S1, S2, S3). The NCBI Conserved Domain Search software allowed us to identify the trans-Isoprenyl Diphosphate Synthase (Trans-IPPS) domain which includes squalene and phytoene synthases. According to the previous domain characterization, wheat PSY3 was classed in the IPSS superfamily and Class I terpene cyclise. The conserved trans-IPPS domain was identified at positions: aa123386, aa112-375 and aa113-376, respectively, for PSY3A, $B$ and D copies. Protein motifs identified based on 

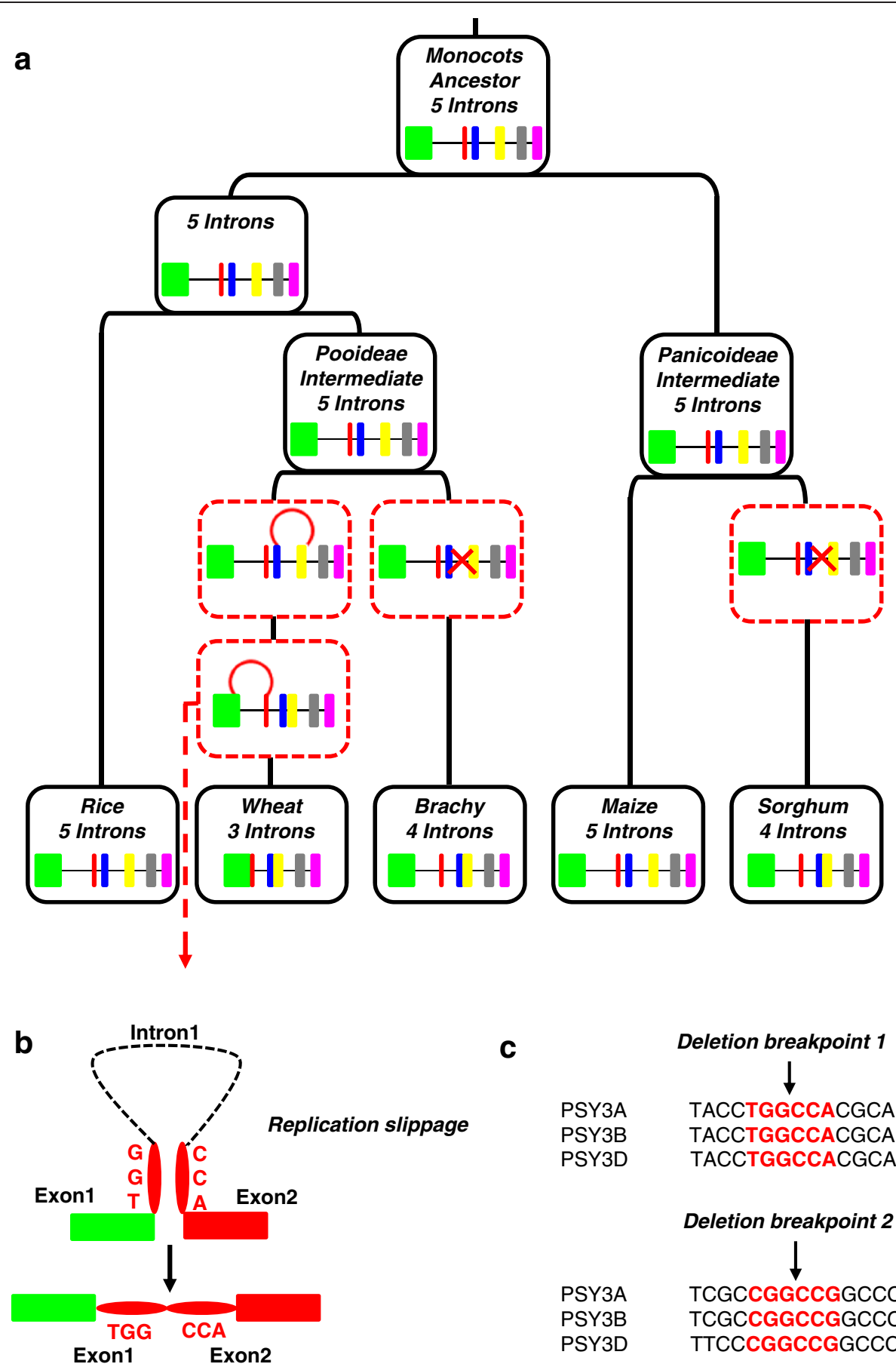

C

PSY3A

PSY3B

PSY3D

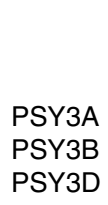

Deletion breakpoint 1

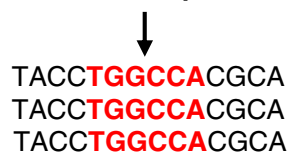

Deletion breakpoint 2

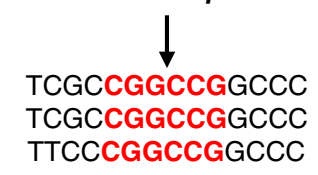

Figure 3 Mechanism driving intron-exon shuffling of PSY3 genes in Grasses. (a) PSY3 gene evolution among Grasses. The modern structure of the PSY3 gene identified in rice, wheat, Brachypodium, maize and sorghum is shown at the bottom with coloured boxes representing conserved exons. The ancestral as well as pooideae and panicoideae PSY3 is structured in five introns and lineage-specific intron losses and consequently exon fusions in wheat, Brachypodium and sorghum are illustrated according to the text description. (b) Representation of intron loss mechanism identified for the wheat PSY3 with inverted and repeated motives that may have driven intron loss through replication slippage via the formation and a DNA loop. (c) Motifs identified at the deletion breakpoints and involved in intron loss due to illegitimate recombination at the splicing site are shown in red.

ScanProsite are available in Additional file 1: Figure S1. ChloroP1.1 [34] identified transit peptides with 56 and 57 residues for PSY3B and PSY3D, but only 6-residues for PSY3A and a signal peptide of two amino acids for Brachypodium PSY3. However, according to ProtComp 9.0 [35] prediction, wheat PSY3 may have a putative 
chloroplast localization. We also analyzed sequence homology between wheat PSY3 proteins: PSY3A and PSY3B showed 91\%, PSY3A and PSY3D 90\% and PSY3B and PSY3D 96\% amino acid sequence identity.

\section{Evolutionary history of PSY gene family}

Throughout monocots, the PSY gene family is characterized by three paralogous genes, annotated as $P S Y 1, P S Y 2$ and $P S Y 3$, while, in eudicots, the presence of $P S Y 1$ and $P S Y 2$ homologs have been reported [5]. The presence of only two copies in eudicots was our starting point to explore evolution of the PSY gene family to explain the presence of a third copy in monocots. We proposed that the angiosperm ancestor was characterized by the presence of the two PSY genes, $P S Y 1$ and PSY2 (identified in monocots such as rice as well as in eudicots such as grape referenced in Figure 4), and a specific evolutionary event must have occurred in the monocots, duplicating PSY1 or PSY2 to give third copy PSY3. To test this hypothesis, we analyzed homology between the three PSY genes of. Brachypodium, rice and sorghum and observed that PSY1 was most closely related to PSY3 in terms of sequence similarity $(>70 \%$ sequence identity at the nucleotide level). However, according to the precise characterization of duplicated chromosomes in monocots reported by Murat et al. [29], we concluded that PSY1-3 genes are not located on chromosomes that have been duplicated during the ancestral shared Whole Genome Duplication (WGD) dating back to 50-70 mya. PSY3 probably originated as a duplication of PSY1 during a more ancient and specific WGD event in monocot evolution that determined its modern localization in monocot genomes (Figure 4). This duplicated region corresponds to a more ancient WGD $(\approx 130$ million years old) that predate the 50-70 million years old ancestral paleotetraploidization in monocots $[36,37]$.

\section{PSY genes expression profile and function investigation in bread wheat}

Since there is no evidence of PSY3 expression in the literature, we used the public wheat Affymetrix chip data [38] based on array hybridization with RNA extracted from wheat grains at different development stages (100 Degree Days (DD), 200DD, 250DD, 300DD, 500DD), from cellular division to starch filling, to unravel the expression of PSY genes in wheat. We blast-searched the PSY3 sequences obtained against Affymetrix chip sequences and found two probes, Ta.20776.1.S1_at and Ta.18880.1.S1_at, homoeologs of $P S Y 1$ and $P S Y 2$, respectively. The expression level of PSY1 in grains was twice that of $P S Y 2$ during all developmental stages except at 500DD when PSY1 levels decreased (Additional file 1: Figure S2). PSY2 expression was constant during grain development, whereas $P S Y 1$ expression exhibited significant differences between 350DD and 500DD ( $\mathrm{p}$-value $=2.22 \mathrm{E}-10$, using the Bonferroni test $)$. Our data showed that PSY1 increased at 200DD while its level remained constant at 300DD and then decreased at 500DD. No evidence was found for PSY3 expression in grain at these developmental stages. To gain insight into PSY gene expression during grain development, we used Single-Strand Conformation Polymorphism (SSCP) analysis [27] to screen wheat RNAs ( $c v$ Recital) extracted from leaves, roots and grains at different development stages. SSCP offered in this experiment the opportunity to (i) detect minor sequence changes in polymerase chain reaction-amplified samples and (ii) evaluate the expression level of three homoeologous copies of each PSY gene in the same running time [38]. In the same PCR reaction, we amplified specifically one paralogous gene and its homoeologous copies that were successfully separated, by capillary electrophoresis according to their sequence differences and assigned to each genome, using amplification profiling approaches on nullitetrasomic lines as references [38]. PSY1 expression was evaluated based on the primer pair PSY1_F1R1 (amplification product of $218 \mathrm{bp}$ ) designed on the fourth exon. Its expression in developing grain (100DD, 200DD, 250DD, 300DD and 500DD) was constant and PSY1-B showed the highest transcript level. PSY1 expression profile did not change in leaves and roots where PSY1-B was always the copy most expressed. We also amplified $P S Y 2$ in each tissue using the primer pair PSY2F1R1, designed on the fourth exon (amplification product of $141 \mathrm{bp).} \mathrm{PSY2} \mathrm{showed} \mathrm{a} \mathrm{constant} \mathrm{expression} \mathrm{pattern} \mathrm{in}$ developing grains, the $\mathrm{B}$ and $\mathrm{D}$ copies showing the highest transcript levels. In leaves, all three homoeolog copies presented the same expression profiles; whereas, in roots, the $\mathrm{D}$ transcript decreased compared to $\mathrm{B}$ and $\mathrm{A}$, whose levels remain unchanged. Using this SSCP approach we were able to detect PSY3 expression using the primer pair PSY3F2R1, designed on the third exon (amplification product of $216 \mathrm{bp}$ ). During grain development at 100DD the D copy showed the highest transcript level, while, at 200DD, amplification intensity of the B copy increased in contrast with the two other homoeologs, which decreased. For the remaining stages, expression levels of the three homoeologous copies were constant. In leaves, A and D copies had the highest expression levels, while in roots, the D copy showed a greater expression than the A and B copies (Additional file 1: Figure S3).

We exploited the wheat PSY3 promoter sequence to search for regulatory elements. Toucan 9.0 workbench software revealed the presence of conserved cis-regulatory elements such as, ABA-responsive element (ABRE), G-box and a Coupling Element (CE), involved in the response to salt and drought stress and application of ABA, and also identified in rice and sorghum $P S Y 3$ promoters $[11,39]$. In order to confirm this hypothesis, Real-Time PCR was used to investigate the expression levels of all three PSYs in leaves and roots during the application of exogenous abscisic acid (ABA). Three concentrations of ABA $(50 \mu \mathrm{M}, 100 \mu \mathrm{M}$ and 


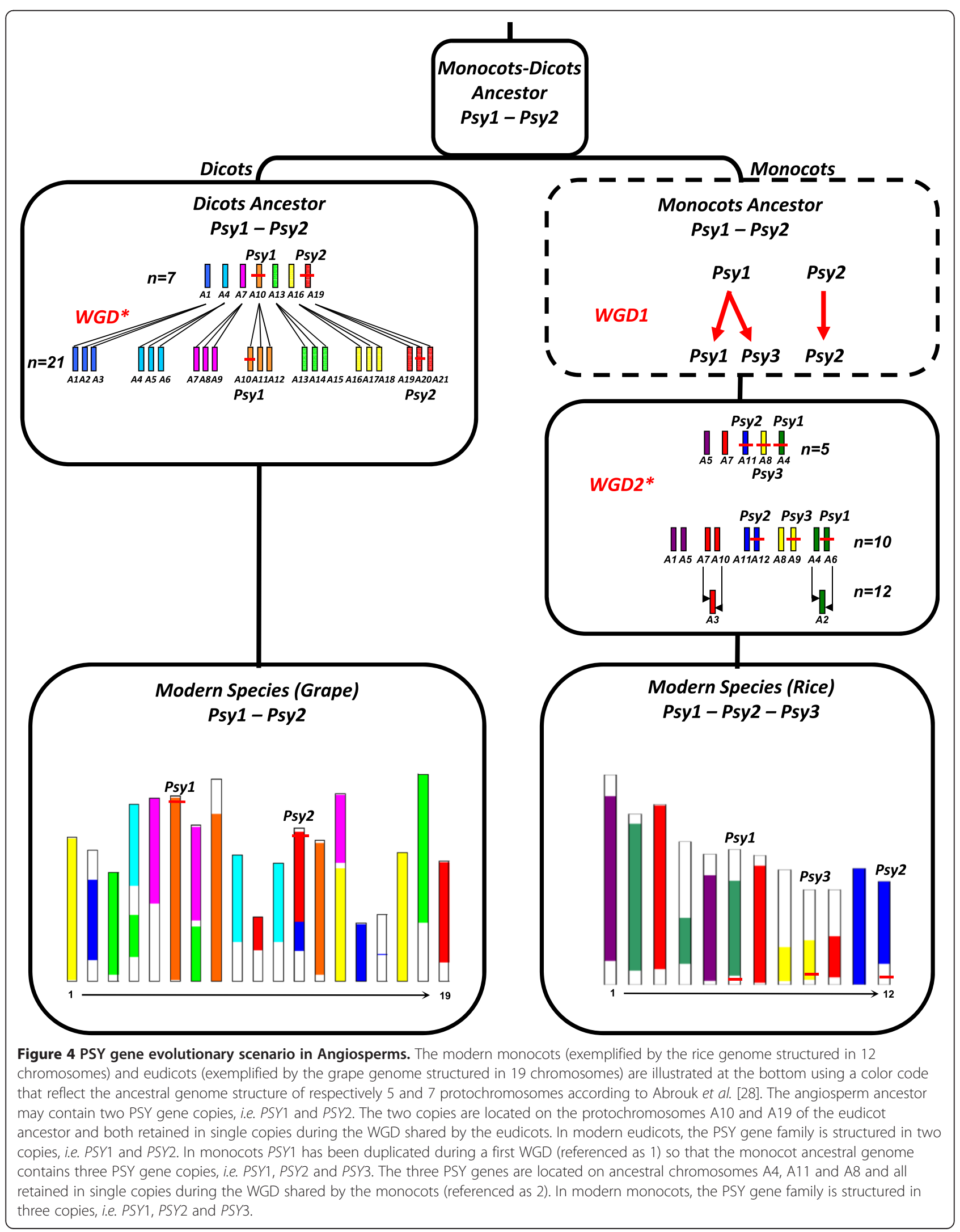


$150 \mu \mathrm{M})$ were tested and samples from each tissue were collected after $0,1,2,5$ and 8 hours (h) of treatment (Figure 5a). In photosynthetically non-stressed tissues, PSY1 and $P S Y 2$ gave the major transcripts, which were three times greater from $P S Y 2$ than from $P S Y 1$ (Figure 5b). In non-stressed roots, PSY1 expression was low and PSY2 expression levels were at least more than 3 times lower than in leaves (Figure 5b). This result agrees with the hypothesis that, in wheat, PSY1 is directly involved in carotenoid accumulation in grain, associated with major QTLs for YP $[13,18,24]$ whereas PSY2 plays a major role in carotenoids accumulation in leaves.

To investigate changes in PSY expression during stress, we determined transcript levels in leaf and root samples after stress treatment. PSY responses to the different ABA concentrations tested were constant. PSY1 mRNA levels, in stressed leaves, underwent to a minimum reduction during the first $2 \mathrm{~h}$ to then returned to the initial level at 5 h. PSY2 transcripts slightly decreased in stressed leaves after $2 \mathrm{~h}$ and then remained constant. PSY3 expression levels increased about 4-fold after $5 \mathrm{~h}$ of stress treatment and then returned to initial levels. In stressed roots, differences in expression levels became more obvious: PSY1 mRNA levels doubled after $5 \mathrm{~h}$ of treatment, while PSY 2 transcript levels doubled after only $2 \mathrm{~h}$ of stress and then decreased gradually after 5 and $8 \mathrm{~h}$ of treatments to initial expression levels (Figure $5 \mathrm{c}$ d-e). PSY3 expression levels started to increase just $1 \mathrm{~h}$ after the ABA application to 18-fold after $2 \mathrm{~h}$ and then returned to lower levels (see Additional file 1: Table S2 for ANOVA analysis). Overall, Figure $5 \mathrm{f}$ clearly shows the effectiveness of ABA treatment on PSY3 up-regulation in roots. Normalized expression data of PSY genes obtained in this experiment are available as Additional file 1: Figure S4.

\section{Discussion}

Carotenoids are widely recognized as essential pigments in plants because of their role in development, photosynthesis, photoprotection and in response to stress since they are essential precursors of apocarotenoids, such as the abscisic acid (ABA). The PSY gene family, playing a central role in carotenoid biosynthesis, is characterized by duplicated gene copies. Their characterization in Grasses and the recent identification of a third copy in rice, maize and sorghum raises questions about PSY gene family organization in wheat. The role and function of PSY1 have been largely investigated because of its association with major QTLs for carotenoid accumulation in wheat grain. Partial sequences of PSY2 from several wheat varieties are available but little is known about its function and, up to now, there was no evidence of a PSY3 homolog and associated activity in wheat. Our current analysis has demonstrated that wheat PSY gene family is characterized by the existence of PSY 1 to 3 gene copies. We report here the precise nature of gene family structure, evolution and function.
Because of the polyploid nature of wheat and the lack of a whole genome sequence, we identified orthologous regions in reference genomes such as Brachypodium and developed COS markers to isolate wheat PSY3 from a BAC library. Wheat PSY3 is characterized by a novel gene structure due to intron loss events leading to four exons and three introns. Rice and maize PSY3, whose gene structure is highly conserved, are characterized by a third intron starting with a non-canonical 5'splicing site GC instead of GT. This type of intron is deleted by the same spliceosome mechanism that removes GT-AG introns, mainly involved in alternative splicing rather than in introns loss events [40]. Brachypodium and sorghum show differences in PSY gene structure, characterized by five exons and four introns. The third intron has been deleted but the causal mechanism is not yet clear. The classical model to explain intron loss is based on recombination of a genomic copy of a gene with a reverse-transcribed copy of a spliced mRNA transcript, deleting one or more adjacent introns. A key aspect of this theory is that only multicellular eukaryotes that have genes transcribed in germ cells would be susceptible to mRNAmediated intron loss [41-45]. We identified inverted and repeated short motifs at deletion breakpoints in the wheat PSY3 sequence, corresponding to the $5^{\prime}$ and the $3^{\prime}$ splicing sites, which may have driven illegitimate recombination leading to the observed intron loss events. Brachypodium and sorghum PSY3 sequences are not characterized by such repeated motifs at deletion breakpoint, suggesting that intron loss occurred by simple genomic deletion. Maize PSY3 intron 2 and rice intron 3 and maize intron 3 and rice intron 2 are similar in size, leading to the hypothesis that these introns may have been inverted but this could not be confirmed due to limited sequence similarities between introns. Howitt et al. [46] described the presence within the intron \#3 in wheat PSY-D1 of an insertion of $1200 \mathrm{bp}$ of an inverted repeat motif. The characterization of either repeated motifs and/or TEs in this intron in Grasses may have driven intron loss or shuffling events leading to the distinct PSY3 gene structures observed.

PSY duplicated genes exist throughout monocots and eudicots. Gallagher et al. [5] proposed an evolutionary model for the Grass PSY gene family in which monocots and eudicots share the same ancestral gene and the same duplication event, giving rise to genes PSY1 and PSY2. Welsch and collaborators [11] proposed that rice $P S Y 3$ originated from PSY2 duplication or vice versa, because of their close similarity in the $5^{\prime} \mathrm{UTR}$ region. We analyzed homology between PSY genes in reference genomes and showed that PSY3 had greater homology with $P S Y 1$ sequence than $P S Y 2$, suggesting that $P S Y 3$ originated from a duplication of $P S Y 1$. Furthermore, public paleogenomics data in Grasses indicated that PSY genes are not located on known duplicated chromosomes originating from the characterized ancestral WGD event 

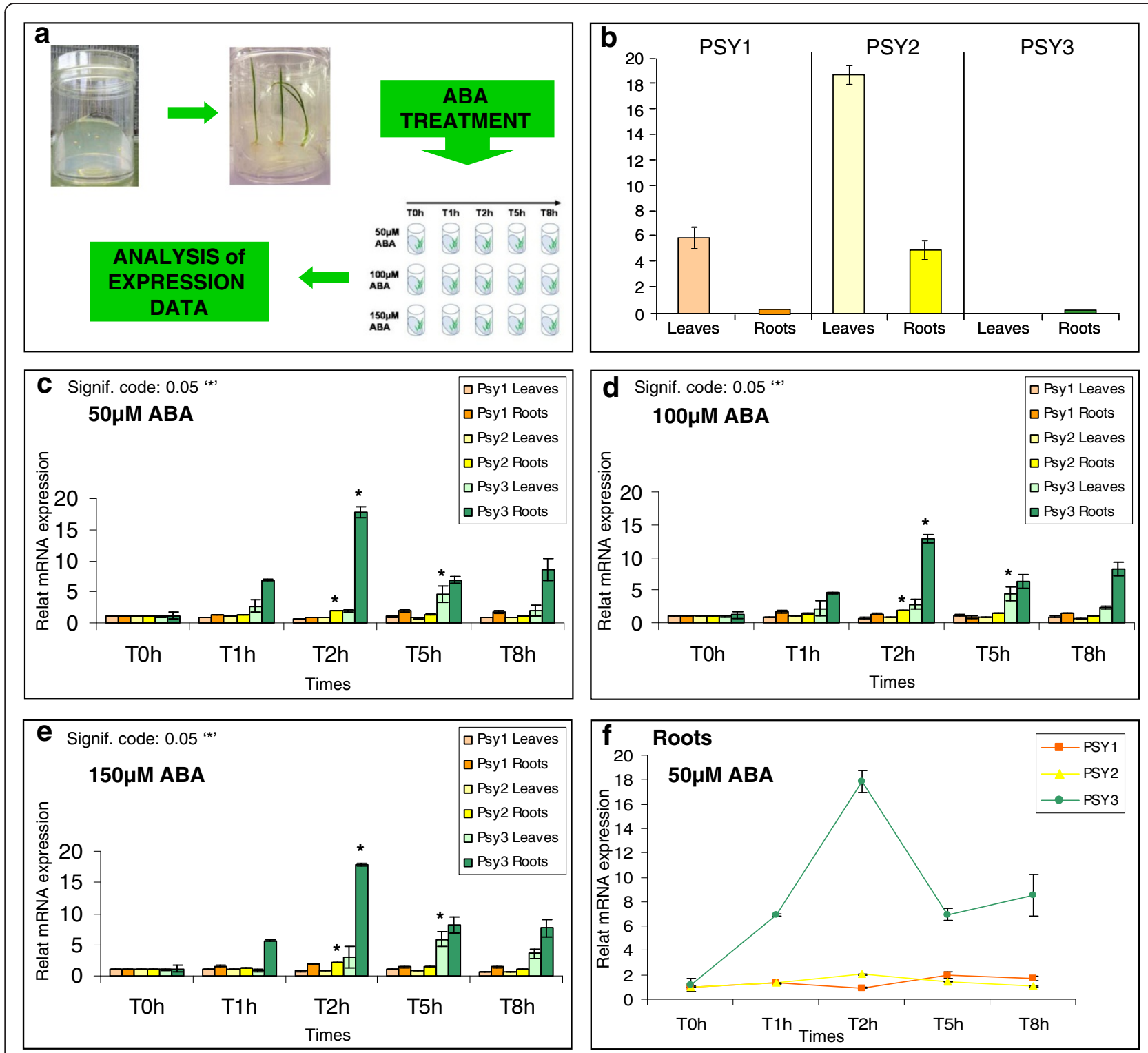

Figure 5 Effect of ABA treatment on wheat PSY expression. (a) Experimental design. (b) Expression level of PSYs in non-stressed leaves and roots. Transcript levels were normalized using the wheat spastin and RNase L inhibitor-like CDNA. (c) (d) (e) Figures show PSY response (y-axis) to ABA treatments at the three different tested concentrations (50 $\mathrm{MM}$ in c panel, $100 \mu \mathrm{M}$ in d panel and $150 \mu \mathrm{M}$ in e panel) in both leaves and roots (see legend) from 0 to 8 hours (x-axis). cDNA and expressed relative to the level detected in not stressed tissues. Each stage has been compared two by two with a confidence level of $95 \%$. The statistical significance in gene expression is shown by an astrisck (*). (f) Efficiency of ABA on PSY3 induction in wheat roots.

reported by Murat et al. [29]. We suggest, according to Jaillon et al. and Tang et al. [36,37], that a more ancient WGD event, specific to monocots has shaped the PSY3 copy as a duplication of PSY1.

Based on a semi-quantitative approach, such as SSCP strategy, we observed PSY expression in wheat grains, leaves and roots. PSY1 and PSY2 homoeologous copies showed stable expression profiles in the different tissues. PSY-B1 was the copy most expressed, while, PSY-B2 and PSY-D2 showed the largest transcript levels in developing grains and, in leaves and roots, PSY-A2 transcripts increased. PSY3 homoeologous copies showed a variable pattern of expression during the first stage of cellular division during early grain development and we found PSY-A3 and PSY-D3 most expressed in leaves and PSY-D3 in roots. However, with this approach, a statistically basis comparison was not possible between the expression levels of the three paralogous genes because it is semi-quantitative and only the broad transcript levels of homoeologous copies of each gene can be compared when amplified with the same primer pairs and in the 
same conditions. Access to recently published Affymetrix data [38] provided the opportunity to investigate PSY gene expression pattern and showed that PSY1 expression level was higher than $P S Y 2$ in developing grains. PSY1 transcripts increased at 200DD to remain constant until 500DD, when they were reduced. In developing maize endosperm, $\mathrm{Li}$ et al. [12] showed that during endosperm carotenogenesis, only PSY 1 transcript increased significantly, starting after 12DAP until 22DAP. Basis on this evidence, we can speculate that wheat $P S Y 1$ in the endosperm follows the same kinetics as the maize PSY1 ortholog and that apparently there is a correspondence in timing of expression between wheat and maize PSY 1 orthologs. Our results are consistent with the evidence that PSY1 plays an important role in carotenoid accumulation in the endosperm and with its association with QTLs for YP on chromosome group 7 in wheat $[13,18,24]$.

Gene expression is closely associated with cis-regulatory DNA sequences (known as cis-elements) located in its upstream regions [47]. In rice and maize, $P S Y 3$ expression is suggested to be strongly inducible in roots by drought and salt stress and application of ABA, since their promoter regions harbour cis-elements involved in plant response to abiotic stress $[11,39]$. We identified several regulatory elements in the wheat PSY3 promoter region, $(i)$ the ABAresponsive element (ABRE) whose core is represented by the G-box, and (ii) a Coupling Element (CE1 or CE3) that is necessary to obtain ABRE functional $[48,49]$. Through a Real-Time PCR approach, we quantified wheat PSY transcript levels in leaves and roots before and after the exogenous application of $\mathrm{ABA}$, observing that $P S Y 2$ represents the most abundant transcript in unstressed leaves, while PSY1 transcript levels were 3-fold lower than in carotenoid accumulation in grains $[13,18,24]$. Wheat PSY2 may thus play an important and complementary role in YP accumulation in photosynthetic tissues. PSY3 expression levels were low in non-stressed leaves compared to PSY1 and PSY2 while its levels increased in non-stressed roots. PSY1 and PSY2 transcript levels did not show significant changes in leaves after ABA treatment. In stressed roots, $P S Y 1$ and $P S Y 2$ levels doubled after $5 \mathrm{~h}$ and $2 \mathrm{~h}$ of treatment respectively. PSY3 showed the most intense response to $\mathrm{ABA}$ as in leaves, after $5 \mathrm{~h}$ of ABA application, $P S Y 3$ transcript was 4fold higher, while in stressed roots after $2 \mathrm{~h}$ its transcript levels increase about 18 -fold. PSY3 was the only gene that showed significant changes of transcript levels in the studied conditions. Carotenoids provide precursors for ABA which is the main apocarotenoid that plants can accumulate under drought conditions, ABA applications having been shown to affect plant growth and development, mimicking the effect of water stress and improving capacity to escape or tolerate such stress [50]. Since PSY genes are considered as rate-limiting in carotenogenesis, we can explain the upregulation of $P S Y 3$ by $A B A$ as the necessity of plants to control carotenoid flux to roots during stress conditions to provide $\mathrm{ABA}$ precursors and thus activate stress responses. In a previous study, Singh et al. [15], studying the association of PSY1-A1 allelic variation with YP colour in durum wheat, speculated about the possible existence of a third PSY on the chromosome 7AL to explain the presence of a second QTL near the marker Xwmc116. We identified the chromosomal bin localization for each homoeolog and the expected syntenic relationships were confirmed assigning PSY3 to chromosome group 5 which has the highest concentration of QTLs and major loci controlling plant adaptation to environment, particularly, those controlling heading date, frost and salt tolerance, whereas a region with a crucial role in drought tolerance is located on chromosome group 7. Multiple-stress QTLs and linked markers have also been detected, suggesting the existence of common mechanisms for different stresses or of gene clusters controlling different stress tolerance processes [51]. For example, in wheat a significant QTL for ABA accumulation in droughted leaves was found on chromosome 5A co-located with the major vernalization responsive gene, $\operatorname{Vrn} 1$ [50], and in the same chromosomal bin we mapped PSY-3A. Aprile et al. [52] studied different physiological reactions to water stress and the substantially different molecular responses of bread and durum wheat genotypes demonstrating that there is a difference between durum and bread wheat response to water stress and that deletion of bin 5AL-10 from Chinese Spring determined a lower response to such stresses. They also showed that the different response by durum and bread wheat can be associated with the absence of $\mathrm{D}$ genome, where genes or factors that modulate expression of cluster of genes may be localized. PSY3 localization and function in wheat are consistent with these data, especially the PSY3D genomic structure, function and expression reported in the current study. Finally, we also showed here that PSY3s are characterized by intron losses, inverted repeats and upregulation by abiotic stresses. It is important to note that biotic/abiotic stresses could also activate transposons and retrotransposons [53] whose expression may have played a role in PSY3 gene structure modification.

\section{Conclusion}

PSY gene family plays an important role in plant development (photoprotection and resistance to stress) as well as is a key contributor to flower and fruit flavours and aromas. The current analysis provides relevant structural (sequence, primers), functional (expression patterns) and evolutionary (gene conservation and specificity) informations that can be used as applied tools in wheat breeding programs.

\section{Methods}

\section{PSY gene orthologs identification}

The methodology used to reassess synteny between wheat/ rice/Brachypodium/sorghum/maize genomes as well as the 
identification of intra-chromosomal duplications in wheat was previously described $[28,29,54,55]$. Briefly, wheat [30,31], rice (41046 genes), Brachypodium (25504 genes), sorghum (34008 genes) and maize (32540 genes) genomes were aligned with the publicly available PSY gene sequence [56] to identify orthologs. Three parameters were used to increase stringency and significance of BLAST sequence alignment by parsing BLASTN results and rebuilding HSPs (high scoring pairs) or pairwise sequence alignments. The first parameter, AL (aligned length), corresponds to the sum of all HSP lengths. The second, CIP (cumulative identity percentage) corresponds to the cumulative percent of sequence identity obtained for all the HSPs (CIP $=\sum$ nb ID by HSP/AL) $x$ 100). The third parameter, CALP is the cumulative alignment length percentage. It represents the sum of the HSP lengths (AL) for all the HSPs divided by the length of the query sequence (CALP $=$ AL/Query length). These parameters allow identification of the best alignment, with the highest cumulative percentage of identity in the longest cumulative length, taking into account all HSPs obtained for any pairwise alignment, regarding PSY sequences. These parameters were applied to all the BLAST alignments that were performed in the present study. Based on the genome-wide synteny analysis, gene relationships between species were then referenced as COS (for conserved gene pairs), $\mathrm{CNV}$ (for tandem duplicated genes) and PAV (for non-conserved genes).

\section{BAC clone screening, sequencing and annotation}

A BAC library from T. aestivum 'Renan' [32] was used in this study, the library has a $6.9 \mathrm{x}$ genome coverage. It consists of 812,544 clones organized in 2592 384-well plates pooled in two steps. The first step aims at mixing the 384 clones of each plate; the second step organizes plate pools in two dimensional matrices. For each 384-well plate, 16line pools and 24-column pools and a total of 40 pools were available. Overall, 274 line and column pools were available to identify BAC clones of interest through PCR screening using PSY primers. Each amplicon (or BAC of interest) in a 384-wells plate was identified by coordinates which identified positive line and column pools in a matrix. By crossing the coordinates of positive pools we determined the plate harbouring the clone of interest. The individual BAC clone of interest was then identified on specific plates by PCR screening. BAC sequencing was performed on the GS Junior (Roche) using the 454 sequencing technology according the procedure described by the manufacturer at the genotyping platform GENTYANE (UMR-1095 GDEC, Clermont-Ferrand).

Genes and repeated elements (TEs) were identified by computing and integrating results based on BLAST algorithms [57], predictor programs and software described below. Gene structure and putative functions were identified by combining results of BLASTN and BLASTX alignments against dbEST [58] and SwissProt databases [59] with the results of predictor program, FgeneSH [60] with default parameters. Known genes were named based on BLASTX results against protein with known functions (SwissProt). Promoter analysis was performed using both TSSP database and Toucan platform [61] to search for cis-elements. PolyA Miner Signal [62] was used in terminator analysis. Characterization of protein functional domain was performed using InterPro [63] and SMART database $[64,65]$, while protein motifs were analysed with ScanProsite [66]. ChloroP1.1 and ProtComp 9.0 were used, respectively, to identify signal peptide and to predict protein subcellular localization.

Transposable elements (TEs) were detected by comparison with two databases of repetitive elements: TREP [67] and Repbase [68]. TEs boundaries were identified with the REPET package [69]. Insertion profile of TEs is identified using a modified version of svg_ltr.pl script [70].

\section{Stress treatment experimental design and quantitative real-time PCR}

Recital seeds were surface sterilized and embryos at 16 days after pollination (DAP) were collected and grown on Murashige and Skoog agar medium with $2 \%$ saccharose and indole-3-acetic acid (IAA). In each pot, three embryos were sown in order to obtain three biological replications for each condition. Plants were grown during the first 3 days in the dark at $25^{\circ} \mathrm{C}$ and were transferred to continuous light at $22{ }^{\circ} \mathrm{C}$ at the two-leaf stage.

To carry out stress experiments, plants were treated with solution of $\mathrm{ABA}$ at different concentration: $50 \mu \mathrm{M}$, $100 \mu \mathrm{M}$ and $150 \mu \mathrm{M}$. Leaf and root samples were collected after $0,1,2,5$ and 8 hours (h) of ABA treatment and were stored at $-80{ }^{\circ} \mathrm{C}$. RNA extraction was performed according to TRIzol ${ }^{\circledR}$ Reagent protocol. DNase set (Qiagen) and RNeasy Minelute Cleanup (Qiagen) kits were used for RNA purification. Integrity and quality of mRNA (contamination assessment of genomic DNA) were checked with the $A_{260} / A_{280}$ ratio with Nanodrop (Thermo Scientific) and on agarose with a classical PCR using a reference gene (see primers below). cDNA synthesis was carried out with a Transcriptor first strand cDNA synthesis kit (Roche) following the procedure described by the manufacturer with $1 \mu \mathrm{g}$ of RNA (cycling conditions: $10^{\prime}$ at $25^{\circ} \mathrm{C} / 30^{\prime}$ at $55^{\circ}$ $\mathrm{C} / 5^{\prime}$ at $85^{\circ} \mathrm{C}^{\circ}$ ). Four primer pairs used for Real-Time PCR analysis (Additional file 1: Table S1) were designed with primer3 in order to obtain exonic specific amplification of $100 \mathrm{pb}$. Real-time PCR was performed using the LightCycler $^{\circledR} 480$ (Roche Diagnostics) and all reactions were performed using the LightCycler ${ }^{\circledR} 480$ DNA SYBR Green I Master (Roche Diagnostics) in $10 \mu \mathrm{l}$ with $4 \mathrm{ng}$ of cDNA and 55 PCR cycles at $60{ }^{\circ} \mathrm{C}$ (hybridation temperature). All samples were prepared in three technical replicates and a negative control using water as a template was included. 
Quantification cycles $(\mathrm{Cq})$ were analysed using the Light Cycler software version 1.5.0 and normalized with two reference genes (wheat spastin (primers: TGCCACTGCCT GATCCAAAT and AGCAAGCCTCTCCAGATCATG) and RNase L inhibitor-like (primers: TTGAGCAACTCATGGA CCAG and GCTTTCCAAGGCACAA ACAT) [71]) using 'Advanced Relative Quantification' module to obtain normalized ratio $\mathrm{E}_{\mathrm{t}}{ }^{-\mathrm{CqT}^{-}} / \mathrm{E}_{\mathrm{r}}{ }^{-\mathrm{CqR}}$ (with $\mathrm{CqT} / \mathrm{CqR}$ : Cycle number at target/reference detection threshold (crossing point) and $\mathrm{E}_{\mathrm{T}} / \mathrm{E}_{\mathrm{R}}$ : Efficiency of target/reference amplification $\left.\left(10^{-1 / \text { slope }}\right)\right)$. Specificity of amplification was confirmed via melting curve analysis of final PCR products by increasing the temperature from $65{ }^{\circ} \mathrm{C}$ to $95{ }^{\circ} \mathrm{C}$. PCR efficiency was calculated for each gene using a standard curve of serial dilutions and used in relative expression analysis. To calculate transcript abundance under stress conditions, the transcript levels were obtained for the controls, at each considered time point and ABA concentration and tissues. All observations were expressed as mean $\pm \mathrm{SD}$; the significance $(P \leq 0.05)$ of variables studied was assessed by one-way analysis of variance (ANOVA) using $\mathrm{R}$ software version 2.13.1.

\section{Additional file}

Additional file 1: S1. PSY3A gene sequences. S2. PSY3B gene sequences. S3. PSY3D gene sequences. Table S1. PSY3 gene primer sets. Table S2. p-value from one-way ANOVA analysis using R software. Figure S1. PSY3 homoeo-alleles protein sequences. Figure S2. PSY1 and PSY2 expression profile in developing grains. Figure S3. PSY3 homoeo-alleles expression during grain development. Figure S4. PSYs expression after ABA treatment.

\section{Competing interests}

The authors declare that there are no competing interests.

\section{Acknowledgements}

This work was supported by grants from the Agence Nationale de la Recherche, Program ANRjc-PaleoCereal (ref: ANR-09-JCJC-0058-01) and Program ANR-Blanc-PAGE (ref: ANR-2011-BSV6-00801). The authors would like also to thank Felicity Vear (INRA Clermont-Ferrand, UMR 1095, France) for her fruitful contribution during the manuscript preparation.

\section{Author details}

'INRA - UMR 1095 'Génétique Diversité Ecophysiologie des Céréales' (GDEC), 5 Chemin de Beaulieu, 63100, Clermont-Ferrand, France. ${ }^{2}$ INRA - Centre National de Ressources Génomiques Végétales (CNRGV), Chemin de Borde Rouge BP 52627, 31326, Castanet Tolosan cedex, France. ${ }^{3}$ DIBCA Department of Agro-Forestry and Enviromental Biology and Chemistry, Sezione di Genetica e Miglioramento Genetico, Via Amendola 165/A, 70126, Bari, Italy.

\section{Authors' contribution}

$\mathrm{BD}$ designed the experiment, performed the analysis and participated in manuscript preparation. FM performed the bioinfomatic analysis and participated in manuscript preparation. $C P, A C, V G, C P, I M, H B$ contributed to the BAC clone screening, sequencing and annotation. CP and PL contributed to the expression analysis. JS and $A B$ designed the research program. JS managed the research group and wrote the article. All authors read and approved the final manuscript.
Received: 9 February 2012 Accepted: 6 June 2012

Published: 6 June 2012

\section{References}

1. Britton G, Liaaen-Jensen S, Pfander H: Carotenoids Handbook. Basel: Birkhäuser Verlag; 2004

2. Lu S, Li L: Carotenoid Metabolism: Biosynthesis, Regulation and Beyond. J of Integrative Plant Biology 2008, 50:778-785.

3. Auldrige ME, McCarty DR, Klee HJ: Plant carotenoid cleavage oxygenases and their apocarotenoid products. Curr Opin Plant Biol 2006, 9:315-321.

4. Fraser PD, Bramley PM: The biosynthesis and nutrional uses of carotenoids. Prog Lipid Res 2004, 43:228-265.

5. Gallagher CE, Matthews PD, Li F, Wurtzel ET: Gene duplication in the carotenoid biosynthetic pathway preceded evolution of the Grasses. Plant Physiol 2004, 135:1776-1783.

6. Cunningham FX, Gantt E: Gene and enzymes of carotenoid biosynthesis in plants. Annu RevPlant Physiol Plant Mol Biol 1998, 49:557-583.

7. Hirschberg J: Carotenoid biosynthesis in flowering plants. Curr Opin Plant Biol 2001, 4:210-218.

8. Ye X, Al-Babili S, Klöti A, Zhang J, Lucca P, Beyer P, Potrykus I: Engineering the pro-vitamin $\mathrm{A}$ ( $\beta$-carotene) biosynthetic pathway into (carotenoidfree) rice endosperm. Science 2000, 287:303-305.

9. Paine JA, Shipton CA, Chaggar S, Howells RM, Kennedy MJ, Vernon G, Wright SY, Hinchliffe E, Adams JL, Silverstone AL, Drake R: Improving the nutritional value of Golden Rice through increased pro-vitamin A content. Nat Biotechnol 2005, 23:429-430.

10. Al-Babili S, Hoa TT, Schaub P: Exploring the potential of the bacterial carotene desaturase $\mathrm{Crtl}$ to increase the beta-carotene content in Golden Rice. J Exp Bot 2006, 57:1007-1014.

11. Welsh R, Wüst F, Bär C, Al-babili S, Beyer P: A Third Phytoene Synthase Is Devoted to Abiotic Stress-Induced Abscisic Acid Formation in Rice and Defines Functional Diversification of Phytoene Synthase Genes. Plant Physiol 2008, 147:367-380.

12. Li F, Vallabhaneni R, Wurtzel ET: PSY3, a new member of the Phytoene Synthase gene famlily conserved in the Poaceae and regulator of abiotic stress - induced root carotenogenesis. Plant Physiol 2008, 146:1333-1345.

13. Pozniak CJ, Knox RE, Clarke FR, Clarke JM: Identification of QTL and association of a phytoene synthase gene with endosperm colour in durum wheat. Theor Appl Genet 2007, 114:525-537.

14. Troccoli A, Borrelli GM, De Vita P, Fares C, Di Fonzo N: Durum wheat quality: a multidisciplinary concept. J Cereal Sci 2000, 32:99-113.

15. Singh A, Reimer S, Pozniak CJ, Clarke FR, Clarke JM, Knox RE, Singh AK: Allelic variation at Psy1-A1 and association with yellow pigment in durum wheat grain. Theor Appl Genet 2009, 118:1539-1548.

16. Patil RM, Oak MD, Tamhankar SA, Sourdille P, Rao VS: Mapping and validation of a major QTL for yellow pigment content on 7AL in durum wheat. Mol Breed 2008, 21:485-496.

17. Cervigni G, Zhang W, Picca A, Carrera A, Helguera M, Manthey F, Miranda R, Dubcosky J, Echenique V: QTL mapping for LOX activity and quality traits in durum wheat. In Proceedings of the $7^{\text {th }}$ international wheat conference SAGPYAV INTA: 27 November-2 December 2005. Argentina: Mar del Plata; 2005.

18. He XY, Zhang YL, He ZH, Wu YP, Xiao YG, Ma CX, Xia XC: Characterization of Phytoene synthase 1 gene (Psy1) located on common wheat chromosome 7A and development of a functional marker. Theor Appl Genet 2008, 116:213-221.

19. Parker GD, Chalmers KJ, Rathjien AJ, Langridge P: Mapping loci associated with flour colour in wheat. Theor Appl Genet 1998, 97:238-245.

20. Mares DJ, Campbell AW: Mapping components of flour and noodle colour in Australian wheat. Aust J Agric Res 2001, 52:1297-1309.

21. Hessler TG, Thomson MJ, Benscher D, Nachit MM, Sorrells ME: Association of a lipoxygenase locus, Lpx-B1, with variation in lipoxygenase activity in durum wheat seeds. Crop Sci 2002, 42:1695-1700.

22. Elouafi I, Nachit MM, Martin LM: Identification of a microsatellite on chromosome $7 \mathrm{~B}$ showing a strong linkage with yellow pigment in durum wheat. Hereditas 2001, 135:255-261.

23. Atienza SG, Ballesteros J, Martin A, Hornero-Mendes D: Genetic variability of carotenoid concentration and degree of esterification among Tritordeum (xTritordeum Ascherson et Graebner) and durum wheat accessions. Agric Food Chem 2007, 55:4244-4251.

24. Zhang W, Dubcovsky J: Association between allelic variation at the Phytoene synthase 1 gene and yellow pigment content in the wheat grain. Theor Appl Genet 2008, 116:635-645. 
25. Kuchel $H$, Langridge $P$, Mosionek $L$, Williams $K$, Jefferies SP: The genetic control of milling yield, dough rheology and baking quality of bread. Theor Appl Genet 2006, 112:1487-1495.

26. Cenci A, Somma S, Chantret N, Dubrovsky J, Blanco A: PCR identification of durum wheat BAC clones containing genes coding for carotenoid biosynthesis enzymes and their chromosome localization. Genome 2004 47:911-917.

27. Quraishi UM, Abrouck M, Bolot S, Pont C, Throude M, Guilhot N, Confolent C, Bortolini F, Praud S, Murigneux A, Chrmet G, Salse J: Genomics in cereals: from genome-wide conserved orthologous set (COS) sequences to candidate genes for trait dissection. Funct Integr Genomics 2009, 9:473-484.

28. Abrouk et al: Paleogenomics of plants: synteny - based modelling of extinct ancestors. Trends Plant Sci 2010, 15:479-487.

29. Murat F, Xu JH, Tannier E, Abrouk M, Guilhot N, Pont C, Messing J, Salse J: Ancestral grass karyotype reconstruction unravels new mechanisms of genome shuffling as a source of plant evolution. Genome Res 2010, 20:1545-1557.

30. Affymetrix [http://www.affymetrix.com/estore/]

31. Wheat Genomics [http://www.cerealsdb.uk.net/

32. INRA - CNRGV [http://cnrgv.toulouse.inra.fr/]

33. Sears ER: Nullisomic - tetrasomic combination in hexaploid wheat. In Chromosomes manipulations and plant genetics. Edited by Riley R, Lewis KR. Edimburgh: Oliver and Boyd; 1966:29-45.

34. ChloroP1.1 Server [http://www.cbs.dtu.dk/services/ChloroP/]

35. Softberry [http://linux1.softberry.com/all.htm]

36. Jaillon $\mathrm{O}$, et al: The grapevine genome sequence suggests ancestral hexaploidization in major angiosperm phyla. Nature 2007, 449:463-467.

37. Tang H, Bowers JE, Wang X, Paterson AH: Angiosperm genome comparisons reveal early polyploidy in the monocot lineage. PNAS 2010, 107:472-477.

38. Pont C, Murat F, Confolent C, Balzergue S, Salse J: RNA-seq in grain unveils fate of neo-and paleopolyploidization events in bread wheat (Triticum aestivum L.). Genome Biol 2011, 12(12):R119.

39. Li F, Tzfadia O, Wurtzel ET: The phytoene synthase gene family in the Grasses. Plant Signaling \& Behavior 2009, 3:208-211.

40. Farrer T, Brock Roller A, Kent WJ, Zahler AM: Analysis of the role of Caenorhabditis elegans GC-AG introns in regulated splicing. Nucleic Acids Res 2002, 30:3360-3367.

41. Roy SW, Gilbert W: The evolution of spliceosomal introns: patterns, puzzles and progress. Nature Review Genetics 2006, 7:211-221.

42. Deng-Ke N, Wen-Ru H, Shu-Wei L: mRNA-Mediated Intron Losses: Evidence from Extraordinarily Large Exons. Mol Biol Evol 2005, 22:1475-1481.

43. Lecharny A, Boudet N, Gy I, Aubourg S, Kreis M: Introns in, intron out in plant gene families: a genomic approach of the dynamics of gene structure. J Structural and Functional Genomics 2003, 3:111-116.

44. Drouin G, Moniz de Sá: Loss of introns in the pollen-specific actin gene subfamily members of potato and tomato. J Mol Evol 1997, 45:509-513.

45. Frugoli JA, MCPeek MA, Thomas TL, McClung CR: Intron loss and gain during evolution of the catalase gene family in Angiosperms. Genetics 1998, 149:355-365.

46. Howitt CA, Cavanagh CR, Bowerman AF, Cazzonelli C, Rampling L, Mimica JL, Pogson BJ: Alternative splicing, activation of cryptic exons and amino acid substitutions in carotenoid biosynthetic genes are associated with lutein accumulation in wheat endosperm. Funct Integr Genomics 2009, 9:363-376.

47. Brivanlou A, Darnell J: Signal transduction and control of gene expression. Science 2002, 295:813-818.

48. Shen $\mathrm{Q}$, Ho TH: Functional dissection of an abscissic acid (ABA) - inducible gene reveals two independent $A B A$ - responsive complexes each containing a G - box and a novel cis - acting element. Plant Cell 1995, 7:295-307.

49. Shen $\mathrm{Q}$, et al: Modular nature of abscissic acid (ABA) response complexes: composite promoter units that are necessary and sufficient for $A B A$ induction of gene expression in barley. Plant Cell 1996, 8:1107-1119.

50. Quarrie SA, Gulli M, Calestani C, Steed A, Marmiroli N: Location of a gene regulation drought-induced abscisic acid production on the long arm of chromosome 5A of wheat. Theor Appl Genetic 1994, 89:794-800.

51. Cattivelli L, Baldi P, Crosatti C, Di Fonzo N, Faccioli P, Grossi M, Mastrangelo AM, Pecchioni N, Stanca AM: Chromosome regions and stress-related sequences involved in resistance to abiotic stress in Triticeae. Plant Mol Biol 2002, 48:649-665.

52. Aprile A, Mastrangelo AM, De Leonardis AM, Galiba G, Roncaglia E, Ferrari F, De Bellis L, Turchi L, Giuliano G, Cattivelli L: Transciptional profiling in response to terminal frought stress reveals differential responses along the wheat genome. BMC Genomics 2009, 10:279.

53. Rapp RA, Wendel JF: Epigenetics and plant evolution. New Phytol 2005, 168:81-91.

54. Salse J, Abrouk M, Murat F, Quraishi UM, Feuillet C: Improved criteria and comparative genomics tool to provide new insights into grass paleogenomics. Briefings in Bioinformatics 2009, 6:619-630.

55. Bolot S, Abrouk M, Quraishi UM, Stein N, Messing J, Feuillet C, Salse J: The 'inner circle' of the cereal genomes. Current Opinion in Plant Biology 2009, 12:119-125.

56. Phytozome [http://www.phytozome.net/]

57. Altschul SF, Gish W, Miller W, Myers EW, Lipman DJ: Basic local alignment search tool. J Mol Biol 1990, 215:403-410.

58. NCBl [http://www.ncbi.nlm.nih.gov/]

59. Expasy [http://expasy.org/sprot/]

60. Salamov A, Solovyev $\mathrm{V}$ : Ab initio gene finding in Drosophila genomic DNA. Genome Res 2000, 10:516-522.

61. Toucan 2 [http://homes.esat.kuleuven.be/ saerts/software/toucan. php\#screenshots]

62. PolyA Miner Signal [http://dnafsminer.bic.nus.edu.sg/PolyA.html]

63. Hunter S, Apweiler R, Attwood TK, Bairoch A, Bateman A, Binns D, Bork P, Das U, Daugherty L, Duquenne L, Finn RD, Gough J, Haft D, Hulo N, Kahn D, Kelly E, Laugraud A, Letunic I, Lonsdale D, Lopez R, Madera M, Maslen J, McAnulla C, McDowall J, Mistry J, Mitchell A, Mulder N, Natale D, Orengo C, Quinn AF, Selengut JD, Sigrist CJ, Thimma M, Thomas PD, Valentin F, Wilson $D, W u C H$, Yeast C: InterPro: the integrative protein signature database. Nucleic Acid Res 2009, 37:224-228

64. Schultz J, Milpets F, Bork P, Ponting CP: SMART, a simple modular architecture research tool: identification of signaling domain. Proc Natl Acad Sci USA 1998, 95:5857-5864.

65. Letunic I, Doerks T, Bork P: SMART 6: recent uploads and developments. Nucleic Acid Res 2009, 37:229-232.

66. De Castro E, Sigrist CJA, Gattiker A, Bulliard V, Langendijk-Genevaux PS, Gasteiger E, Bairoch A, Hulo N: ScanProsite: detection of PROSITE signature matches and ProRule-associated functional residues in proteins. Nucleic Acids Res 2006, 34:W362-W365.

67. Graingenes: A database for Triticeae and Avena [http://wheat.pw.usda.gov./ ITMI/Repeats/]

68. Jurka J: Repbase update: a database and an electronic journal of repetitive elements. Trends Genet 2000, 9:418-420.

69. Flutre T, Duprat E, Feuillet C, Quesneville H: Considering transposable element diversification in de novo annotation approaches. PLoS One 2011 6(1):e16526.

70. Kronmiller BA, Wise RP: TEnest: Automated Chronological Annotation and Visualization of Nested Plant Transposable Elements. Plant Physio/ 2008, 146:45-59.

71. Giménez MJ, Pistón F, Atienza SG: Identification of suitable reference genes for normalization of qPCR data in comparative transcriptomics analyses in the Triticeae. Planta 2011, 233:163-173.

doi:10.1186/1471-2164-13-221

Cite this article as: Dibari et al:: Deciphering the genomic structure, function and evolution of carotenogenesis related phytoene synthases in grasses. BMC Genomics 2012 13:221.

\section{Submit your next manuscript to BioMed Central and take full advantage of:}

- Convenient online submission

- Thorough peer review

- No space constraints or color figure charges

- Immediate publication on acceptance

- Inclusion in PubMed, CAS, Scopus and Google Scholar

- Research which is freely available for redistribution 